Cite this: Analyst, 2013, 138, 6073

Received 24th May 2013

Accepted 13th August 2013

DOI: $10.1039 /$ c3an01041d

www.rsc.org/analyst

\section{A GFP-tagged nucleoprotein-based aggregation assay for anti-influenza drug discovery and antibody development $\uparrow$}

\author{
Helma Antony ${ }^{\mathrm{abc}}$ and Patrick M. Schaeffer ${ }^{\star a b c}$ \\ Influenza is a viral pandemic that affects millions of people worldwide. Seasonal variations due to genetic \\ shuffling and antigenic drifts in the influenza viruses have necessitated continual updating of therapeutics. \\ The growing resistance to current influenza drugs has increased demand for new antivirals. The highly \\ conserved nature of NP, a multi-functional viral protein that is serotypically distinct and abundantly \\ expressed during infection, has led to its use in developing universal biotherapeutics and vaccines that \\ could be effective against the virus, irrespective of its strain variations. Compounds causing aggregation \\ of NP have recently been shown to be potent antivirals but require the development of new high- \\ throughput assays capable of screening compounds with similar modes of action. Here, we describe the \\ development of a new bioassay for the Influenza A nucleoprotein (NP). The assay was developed to \\ quantify ligand-induced aggregation of a GFP-tagged NP and was validated with aggregation-inducing \\ compounds such as nucleozin and a NP-specific antibody. The new NP-GFP aggregation assay can be \\ performed with partially purified or mixtures of proteins and is amenable to a high-throughput format. \\ Using this assay, we demonstrate the potential of a new anti-NP polyclonal antibody that we have \\ obtained from chicken. This cost-effective high-yield source of anti-NP IgY has potential for large-scale \\ production and development of therapeutic antibodies. The simplicity, speed and flexibility of this assay \\ make it an invaluable tool for timely development of effective antivirals that can help to control future \\ epidemics.
}

\section{Introduction}

Despite years of research into influenza, this long-known, highly contagious disease continues to take its toll on millions of people of all ages every year. A substantial proportion of the affected suffer the life-threatening variant resulting in a typical mortality of 250 000-500 000 per flu season. ${ }^{1,2}$ The major factors impeding the development of efficient antivirals are: (1) the growing resistance of current influenza strains against existing drugs; and (2) the continued evolution of the influenza virus. ${ }^{2,3}$ Both of these generate strains with varying virulence and transmissibility each season. The key molecular event responsible for this is the genetic reassortment or gene shuffling that occurs in the course of a viral infection. During viral RNA replication in a host cell infected with multiple influenza

${ }^{a}$ Centre for Biodiscovery and Molecular Development of Therapeutics, James Cook University, Douglas, QLD 4811, Australia.E-mail: patrick.schaeffer@jcu.edu.au

${ }^{b}$ Comparative Genomics Centre, School of Pharmacy and Molecular Sciences, James Cook University, Douglas, QLD 4811, Australia

${ }^{c}$ School of Pharmacy and Molecular Sciences, James Cook University, Douglas, QLD 4811, Australia

$\dagger$ Electronic supplementary information (ESI) available. See DOI:

strains, segments of the viral RNA genome get shuffled randomly (genome reassortment). The newly assembled viral progeny may contain mutations that empower the new strains with properties like drug-resistance or evasion of pre-existing host immunity ( $c f$. 'antigenic drift'). For example, the 2009 H1N1 pandemic strain (H1N1pdm09 S-OIV), which is a reassortant progeny of the avian, human, and swine influenza viruses ${ }^{4}$ was found to be resistant to oseltamivir [Tamiflu] in patients with prior exposure to the drug due to accumulation of mutations in the drug-binding site., ${ }^{5,6}$ Therefore, there is a crucial need to develop antivirals that can surpass the seasonal variation and drug-resistance issues in order to be efficient. Current influenza drugs are comprised of neuraminidase inhibitors (oseltamivir/Tamiflu, zanamivir/Relenza, peramivir and laninamivir) and adamantine derivatives (amantadine and rimantadine) that exclusively target the viral envelope and membrane proteins, neuraminidase (NA) and M2 hydrogen ion channel, respectively. ${ }^{2,3}$ Both these proteins are highly prone to mutations and thus develop drug resistance rapidly. ${ }^{2,3}$ Furthermore, the only approved prophylactic measure currently available employs immunotherapy using neutralising antibodies that target the mutation-prone envelope protein haemagglutinin (HA). ${ }^{7}$ As a result of seasonal variation in the HA 
protein, these vaccines are continually reformulated and annually re-administered to the public at a great expense. Even then, these vaccines do not protect against novel 'antigenicshift' subtypes that have evolved with changes in the entire HA gene, and thus posing pandemic risks such as the H3 subtype in the 1968 pandemic.

NP is a biologically significant multi-functional protein critical for viral RNA replication, genome trafficking and viral assembly. ${ }^{8}$ More importantly, NP is serotypically distinct ${ }^{8}$ and extensively conserved among Influenza A strains. ${ }^{8-10}$ The evolution rate of the influenza viral genes is estimated to be $2 \times$ $10^{6}$ higher than that of eukaryotic gene equivalents, ${ }^{11}$ with an average nucleotide substitution rate of up to $4.6 \times 10^{-3}$ substitutions per site per year in HA genes, ${ }^{12}$ and up to $4.4 \times$ $10^{-3}$ substitutions per site per year in NA genes. ${ }^{\mathbf{1 3 , 1 4}}$ In comparison, the NP gene has only $2.4 \times 10^{-3}$ substitutions per site per year according to a recent large-scale analysis of $>5000$ NP nucleotide sequences. ${ }^{15}$ At the protein level, only $30.1 \%$ amino acid residues were found to be polymorphic in NP, out of the 2500 Influenza A NP sequences analysed. ${ }^{10}$ Furthermore, NP is abundantly expressed during infection ${ }^{\mathbf{1 6}}$ and readily detected as early as 2 days post-infection in mouse models. ${ }^{17}$

Recently, several groups have investigated the potential of NP as an antiviral target and have identified a few prospective drugs - i.e. nucleozin and its analogues - that cause aggregation of NP, thereby blocking its functions, such as viral genome trafficking ${ }^{18}$ and replication. ${ }^{19-22}$ These compounds were shown to be effective against a broad range of Influenza A viral strains including $\mathrm{H} 1 \mathrm{~N} 1, \mathrm{H} 3 \mathrm{~N} 2$, and $\mathrm{H} 5 \mathrm{~N} 1$ in cell culture experiments and against highly pathogenic avian influenza (HPAI) H5N1 in mouse models. ${ }^{19-22}$ However, in view of the evolution of strain variants and drug resistance, there is also a need to identify NP mutants that are likely to be resistant to these drugs.

Owing to its ability to confer heterosubtypic immunity, antiNP antibodies have recently received increasing emphasis in developing a universal vaccine. Thus far, vaccinations with purified NP or DNA vectors have been the main mode of eliciting the antibody response. ${ }^{23-26}$ The potential of non-neutralising anti-NP antibodies that are elicited by natural infection and these vaccinations are shown to play an important role in conferring heterosubtypic immunity. ${ }^{27}$ Furthermore, passive administration of anti-NP antibodies to naive mice has not only been shown to convey protection against infection, ${ }^{27}$ but also rescues the reduced heterosubtypic immunity up to 1.5 years post-infection..$^{28}$ Nevertheless, it was noted that mice required an injection of purified anti-NP IgG to a final serum titer of $10^{5}$ in order to reduce the viral load. ${ }^{17}$ Large-scale production of anti-NP antibodies for passive administration would thus be an ideal strategy to boost antibody titers.

In birds, the circulating serum antibodies are transferred to egg yolks as a means of conferring passive immunity to the embryos. $^{29}$ Chicken immunoglobulin (IgY) is very similar to mammalian IgG and highly stable. ${ }^{30-34}$ IgY has been used successfully for therapeutic and prophylactic administration in humans and animals with good tolerance and no complement activation. ${ }^{35,36}$ Passive administration of IgY can be accomplished via oral or intranasal routes. ${ }^{37,38}$ In recent years, this non-invasive technology is being increasingly utilised as a cheap source of antibody production owing to its high and longlasting yields (up to 2 years). ${ }^{39}$ A typical egg laying hen can yield $1500 \mathrm{mg}$ IgY per month, of which $2-10 \%$ is antigen-specific IgY. ${ }^{40}$ As IgYs are the only antibody isotype present in the egg yolk, extraction is fast and simple. Compared to other sources of antibody production, hyperimmunised chickens often produce antibodies against highly conserved epitopes that may not normally be recognised by mammalian immune systems; ${ }^{37}$ thereby increasing the chances of obtaining a higher affinity antibody against the desired antigen. Passive administration of polyclonal IgY generated against $\mathrm{H} 5 \mathrm{~N} 1$ and $\mathrm{H} 1 \mathrm{~N} 1$ viruses has previously been shown to be efficacious in conferring protection against homologous and heterologous lethal challenge infection in mice. ${ }^{37,41} \mathrm{~A}$ further study has demonstrated the in vitro antiviral activity of anti-Influenza B IgY. ${ }^{42}$

Here, we report for the first time the production of a highly specific polyclonal anti-NP IgY from chicken egg yolks that has potential for immunotherapy and the development of a simple and fast aggregation-based in vitro assay (Fig. 1) capable of identifying NP-targeting biologics and chemicals with a similar

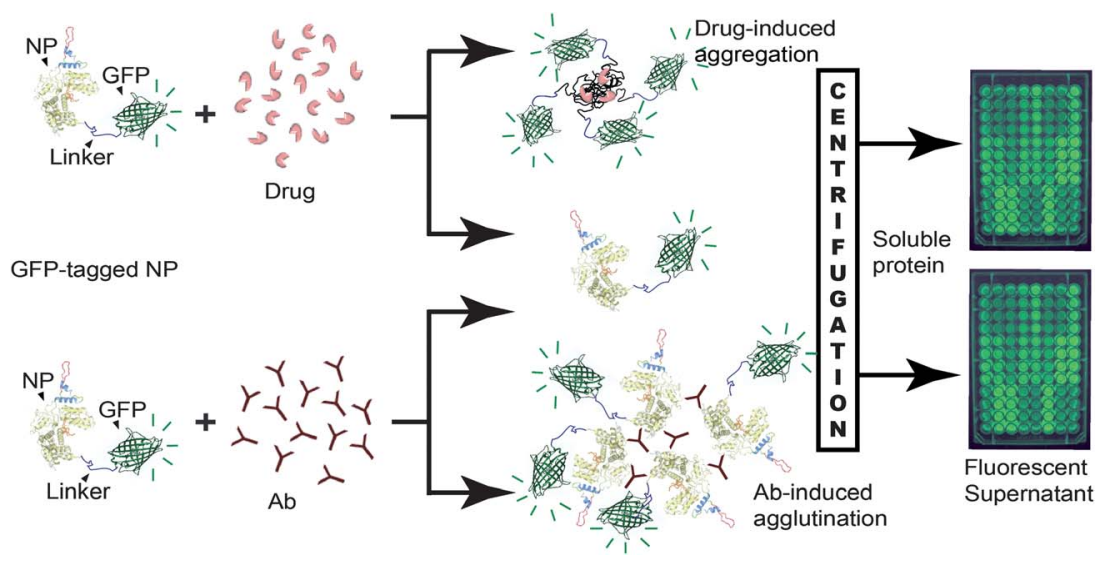

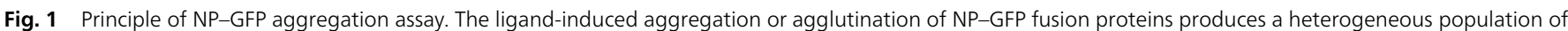

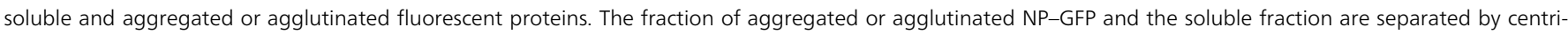
fugation and measured by fluorimetry. Ab: antibody. 
mode of action to that of nucleozin, i.e. specific aggregation of NP. ${ }^{19-22}$

\section{Materials and methods}

\section{Reagents}

The H1N1 NP purified from A/Beijing/262/95 H1N1 influenza virus infected chicken eggs was obtained from Virusys Corporation, Taneytown, MD. Nucleozin was obtained from ChemBridge Corporation, San Diego, CA.

\section{Cloning}

Escherichia coli strain DH12S was used routinely as the host strain during construction of vectors. The NP used in this study was originally derived from pGEM-NP containing the NP sequence of A/Viet Nam/1203/2004 (H5N1) (GenBank:AAW80720). ${ }^{43}$ To generate pET-His $_{6}-\mathrm{NP}$ (pHA228), the pET-His ${ }_{6}-\mathrm{NP}-\mathrm{GFP}^{43}$ was digested with NheI, end-filled and re-ligated to introduce a stop codon at the end of the NP open reading frame, thereby eliminating the C-terminal GFP coding sequence. The vector pET16bD2 encoding the domain III of the Dengue (DENV-2 TSV01) virus envelope protein (EDIII) with an N-terminal 6-His-tag ${ }^{44,45}$ was a kind gift from A/Prof. Subhash Vasudevan (Duke-NUS, Singapore).

\section{Protein expression and purification}

Vectors were transformed into the $E$. coli strain BL21(DE3)RIPL (Stratagene) for protein expression. NP-GFP ${ }^{43}$ and NP were expressed and purified using Profinity IMAC Ni-charged resin (Bio-Rad) as described previously. ${ }^{46}$ Briefly, cultures were grown at $37{ }^{\circ} \mathrm{C}$ in OvernightExpress Instant TB Medium (Novagen) until an $\mathrm{OD}_{600}$ of 0.5 was reached, followed by incubation at $16{ }^{\circ} \mathrm{C}$ with vigorous shaking for 3 days. Harvested cells were resuspended in lysis buffer 1 ( $45 \mathrm{mM} \mathrm{Na}_{2} \mathrm{HPO}_{4}, 5 \mathrm{mM} \mathrm{NaH}_{2} \mathrm{PO}_{4}$ (pH 7.8), $300 \mathrm{mM} \mathrm{NaCl,} \mathrm{10 \%} \mathrm{(v/v)} \mathrm{glycerol,} 2 \mathrm{mM} \beta$-mercaptoethanol, $10 \mathrm{mM}$ imidazole), lysed at $10000 \mathrm{psi}$ using a French pressure cell and centrifuged at $40000 \mathrm{~g}$ for $30 \mathrm{~min}$ at $4{ }^{\circ} \mathrm{C}$. Eluted fractions containing NP and NP-GFP in elution buffer 1 (lysis buffer $1+200 \mathrm{mM}$ imidazole) were precipitated at $4{ }^{\circ} \mathrm{C}$ with 0.3 and $0.5 \mathrm{~g} \mathrm{ml}^{-1}\left(\mathrm{NH}_{4}\right)_{2} \mathrm{SO}_{4}$, respectively and centrifuged at $18000 \mathrm{~g}$ for $30 \mathrm{~min}$ at $4{ }^{\circ} \mathrm{C}$. GFP, ${ }^{46} \mathrm{NP}$ and NP-GFP pellets were resuspended and stored in buffer $\mathrm{A}\left(45 \mathrm{mM} \mathrm{Na} \mathrm{HPO}_{4}, 5 \mathrm{mM}\right.$ $\mathrm{NaH}_{2} \mathrm{PO}_{4}$ (pH 7.8), $300 \mathrm{mM} \mathrm{NaCl}, 10 \%$ (v/v) glycerol) and buffer B (45 mM Na $2 \mathrm{HPO}_{4}, 5 \mathrm{mM} \mathrm{NaH}_{2} \mathrm{PO}_{4}$ (pH 7.8), 10\% (v/v) glycerol, $2 \mathrm{mM} \beta$-mercaptoethanol), respectively. GFP was stored in buffer B. For aggregation studies with nucleozin, the NP-GFP, GFP and NP proteins were dialysed with buffer C (50 mM Tris$\mathrm{Cl}$ (pH 7.5), $100 \mathrm{mM} \mathrm{NaCl}$ ) consistent with previous studies. ${ }^{\mathbf{1 9 , 4 7}}$

For the control viral protein EDIII: following transformation with pET16bD2, E. coli BL21(DE3)RIPL cells were grown at $16{ }^{\circ} \mathrm{C}$ with vigorous shaking $(200 \mathrm{rpm})$ in OvernightExpress Instant TB Medium (Novagen) for 4 days. Cells were lysed and centrifuged as above. The pellet containing EDIII was refolded from inclusion bodies using a modification of a previously described protocol. ${ }^{44}$ Briefly, the pellet was washed in $1 \mathrm{M}$ urea and $2 \%$ Triton X-100, centrifuged at $40000 \mathrm{~g}$ for $30 \mathrm{~min}$ at $4{ }^{\circ} \mathrm{C}$, and solubilised in lysis buffer $2\left(18 \mathrm{mM} \mathrm{Na}_{2} \mathrm{HPO}_{4}, 2 \mathrm{mM} \mathrm{NaH} \mathrm{PO}_{4}\right.$ (pH 7.8), $300 \mathrm{mM} \mathrm{NaCl,} 8 \mathrm{M}$ urea, $10 \mathrm{mM} \beta$-mercaptoethanol, final $\mathrm{pH}$ 8.25) overnight. The soluble fraction was loaded onto a Profinity IMAC Ni-charged resin (Bio-Rad) pre-equilibrated with the same buffer. Proteins were eluted with elution buffer 2 (lysis buffer $2+200 \mathrm{mM}$ imidazole) and refolded by dialysis (10 000 MWCO SnakeSkin ${ }^{\circledR}$ pleated dialysis tubing, regenerated-cellulose, Pierce) against MilliQ water $(1 \mathrm{l} \times 2)$ for $28 \mathrm{~h}$ at $4{ }^{\circ} \mathrm{C}$.

All purified proteins were assessed for purity by SDS-PAGE and quantified by standard Bradford assay (Sigma).

\section{Production and purification of IgY}

Female leghorn chickens Gallus gallus were repeatedly immunised (i.e. 6 boosters following primary) via intramuscular injections of $100 \mu \mathrm{g}$ purified NP mixed $1: 1(\mathrm{v} / \mathrm{v})$ with adjuvant (Emulsigen, Intervet). As a negative control, another set of chickens was repeatedly immunised with purified EDIII mixed with adjuvant. Eggs were collected 3-4 weeks post-immunisation and the IgY was extracted and purified from the yolks using modified PEG precipitation and dialysis. ${ }^{48}$ Briefly, the yolk was separated from the egg whites and the IgYs in the yolk were purified by a 2-step precipitation with PEG 6000 at a final concentration of $12 \%(\mathrm{w} / \mathrm{v})$. Following precipitation, the IgY extract was dialysed in PBS. IgYs were assessed for purity by SDS-PAGE (reducing and non-reducing) and quantified by standard Bradford assay (Sigma).

\section{Immunoblotting}

Samples of purified recombinant proteins separated by SDSPAGE were transferred onto PVDF membrane (BioRad) pre-wet with methanol for $30 \mathrm{~s}$, via semi-dry electroblotting at $15 \mathrm{~V}$ for $25 \mathrm{~min}$ in transfer buffer (0.19 M glycine, $25 \mathrm{mM}$ Tris $)$. Following blocking with $5 \%$ skim milk in PBS buffer $(10 \mathrm{mM}$ $\mathrm{Na}_{2} \mathrm{HPO}_{4}, 1.5 \mathrm{mM} \mathrm{NaH} \mathrm{PO}_{4}$ (pH 7.4), $137 \mathrm{mM} \mathrm{NaCl}, 2.7 \mathrm{mM}$ $\mathrm{KCl}$ ) at RT for $1 \mathrm{~h}$, blots were probed with diluted (1 : 1000 in $1 \%$ skim milk in PBS buffer) anti-NP or anti-EDIII IgYs at RT for $1 \mathrm{~h}$. After washing three times for 5 min with PBS-T buffer (PBS + $0.05 \%(\mathrm{v} / \mathrm{v})$ Tween-20), blots were probed with peroxidaseconjugated anti-IgY (Jackson Immunoresearch Laboratories Inc.) diluted $1: 5000$ in 1\% skim milk in PBS buffer for $1 \mathrm{~h}$ at RT. PVDF membranes were washed again three times with PBS$\mathrm{T}$ buffer and developed with $5 \mathrm{ml}$ SIGMAFAST ${ }^{\mathrm{TM}}$ 3,3'-diaminobenzidine $/ \mathrm{H}_{2} \mathrm{O}_{2}$ solution to visualise immunoreactive bands.

\section{NP-GFP aggregation assay with nucleozin}

For the aggregation reactions, 9 volumes of NP, NP-GFP and GFP proteins in buffer $\mathrm{C}$, were treated individually or as mixtures, with 1 volume of increasing concentrations of nucleozin in DMSO and incubated at room temperature (RT, $25{ }^{\circ} \mathrm{C}$ ) for $1 \mathrm{~h}$. Reactions were performed in a total reaction volume of $75 \mu \mathrm{l}$ and at a final concentration of $4 \mu \mathrm{M}$ NP. After incubation, reactions were centrifuged at $15000 \mathrm{~g}$ for $30 \mathrm{~min}$ at $4{ }^{\circ} \mathrm{C}$ in a bench-top centrifuge (Eppendorf 5810R, rotor: F45-48PCR). Quantification was carried out using densitometric 
analysis and/or by measuring the fluorescence in the supernatant as previously described. ${ }^{\mathbf{4 3 , 4 9}}$

Briefly, for densitometric analysis, $10 \mu \mathrm{l}$ of supernatant was subjected to SDS-PAGE followed by Coomassie blue staining. Protein bands were integrated using ImageJ software (http:// rsbwed.nih.gov/ij/) and normalised against bands obtained for control (untreated) reactions as previously described. ${ }^{49}$ For fluorescence measurements, $50 \mu \mathrm{l}$ supernatants were transferred to a black 96-well plate (Nunclon) and read in a fluorescence plate reader (Victor V Wallace Perkin-Elmer) with $40 \mathrm{~nm}$ band-width filters at excitation and emission settings of $355 \mathrm{~nm}$ and $535 \mathrm{~nm}$, respectively.

Soluble fractions $\left(F_{\text {sol }}\right)$ were calculated by dividing the fluorescence value obtained for a reaction (+nucleozin) with the fluorescence value of a control reaction (-nucleozin).

\section{NP-GFP agglutination assay with IgY}

NP-GFP was mixed $1: 1(\mathrm{v} / \mathrm{v})$ with varying concentrations of IgY (in PBS) in 30-90 $\mu \mathrm{l}$ reaction volumes and incubated at RT for 30 min. The final concentration of NP-GFP was $0.5 \mu \mathrm{M}$ in the reaction volume, except for the concentration-dependent agglutination where varying concentrations of NP-GFP were used as specified (Fig. 3E). After incubation, reactions were centrifuged at $15000 \mathrm{~g}$ for $30 \mathrm{~min}$ at $4{ }^{\circ} \mathrm{C}$. The pellets were analysed by SDS-PAGE and silver staining. ${ }^{50}$ Densitometric quantification of the stained protein bands and the fluorescence in the supernatants were determined as above.

\section{Results}

\section{Principle of NP-GFP aggregation assay}

We have previously developed an assay called GFP-Basta to measure the ligand-induced thermal stabilization of a protein of interest. ${ }^{49}$ We applied this assay on NP-GFP and identified an NP-binding ssDNA that was further applied to develop an ultrasensitive immuno-PCR assay. ${ }^{43}$ A further assay based on differential scanning fluorimetry of GFP-tagged proteins ${ }^{51}$ was recently applied to NP-GFP, but the intrinsic high stability of NP was a limiting factor for this assay and could therefore not be applied for high-throughput screening of stabilizing compounds. Building on these previous observations and limitations, a new NP-GFP aggregation assay was developed to allow identification of NP-targeting anti-influenza agents with similar modes of action to that of nucleozin, i.e. irreversible and specific aggregation of NP. ${ }^{19-22}$ The NP-GFP aggregation assay uses GFP tethered to the C-terminus of NP as a quantitative reporter of NP aggregation and is applied for the detection of chemically-triggered NP aggregation at room temperature (Fig. 1). Following treatment of NP-GFP with molecules triggering aggregation of NP in a 96-well PCR plate, the reaction is centrifuged and the soluble fraction $\left(F_{\text {sol }}\right)$ of NP can be directly determined from the fraction of residual fluorescence corresponding to the fraction of soluble NP-GFP in the supernatant. The quantification can be performed by fluorimetric analysis using a fluorescence plate reader or by SDS-PAGE and densitometric analysis of protein bands.

\section{Validation of NP-GFP aggregation assay with nucleozin}

Nucleozin inhibits NP functionality by aggregating NP molecules into high-order oligomers. ${ }^{19,21}$ To validate the utility of NP-GFP for developing an aggregation-based drug screening assay, we tested and compared the effect of nucleozin on NPGFP, GFP and NP. Nucleozin and protein samples were mixed and incubated at room temperature for 1 hour. Thereafter, the protein aggregates were removed by centrifugation and the soluble fractions of each protein was quantitated by SDS-PAGE and densitometry (Fig. 2A). In order to eliminate the effect of buffer variation and allow direct comparison of specific druginduced effects on the different proteins in identical conditions, we first treated a mixture of GFP $(4 \mu \mathrm{M})$, NP $(2 \mu \mathrm{M})$ and NP-GFP $(2 \mu \mathrm{M})$ with increasing concentrations of nucleozin $(2,4,20$ and $40 \mu \mathrm{M}$ ) for $1 \mathrm{~h}$. Comparison of the aggregation curve of NP with that of NP-GFP immediately suggested that nucleozin aggregates NP slightly more efficiently than NP-GFP (Fig. 2B). Nevertheless, the data also demonstrated that a similar concentration-dependent aggregation was triggered by nucleozin in both NP and NP-GFP, while GFP remained unaffected across all nucleozin concentrations (Fig. 2B). An unidentified protein (CP) that co-purified with NP was also observed to be

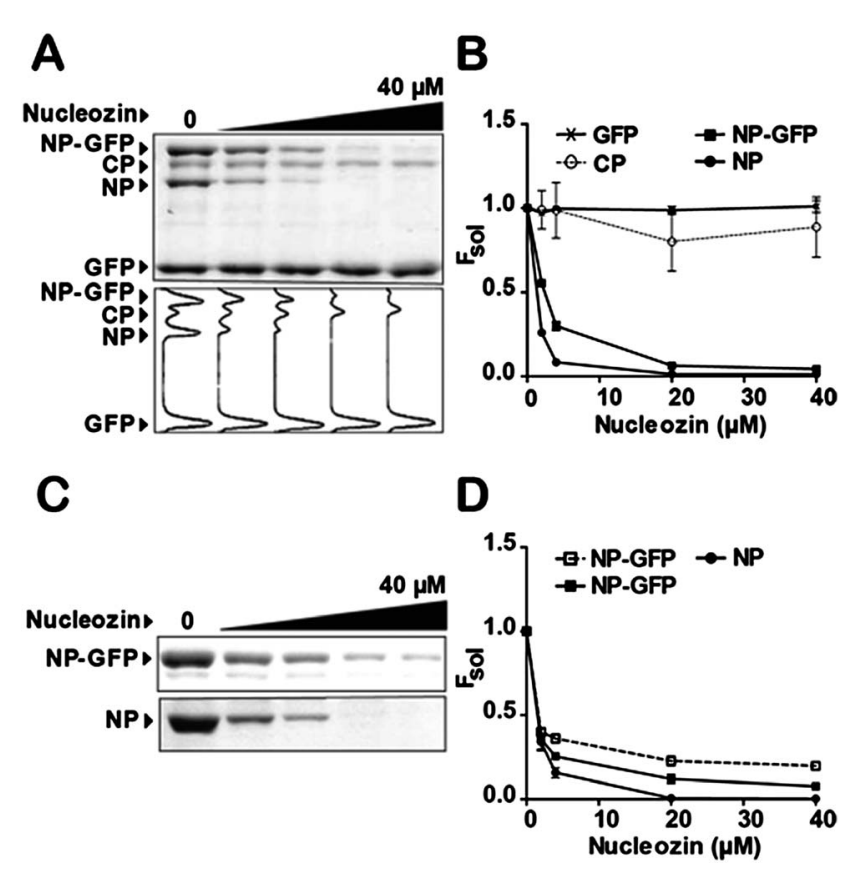

Fig. 2 Quantitative determination of nucleozin-induced NP aggregation. (A) SDS-PAGE of the supernatants of a NP, NP-GFP and GFP protein mixture treated with increasing concentrations $(2,4,20,40 \mu \mathrm{M})$ of nucleozin at RT for $1 \mathrm{~h}$ (top panel) and the corresponding electropherogram generated by ImageJ analysis (bottom panel). CP is an unidentified protein that co-purified with NP. (B) Aggregation curves obtained by densitometric analysis of NP, NP-GFP and GFP protein mixture treated with increasing concentrations of nucleozin at RT for $1 \mathrm{~h}$ ( $n=3$, see Table S1 in ESI + ). (C) SDS-PAGE of the supernatants of NP and NP-GFP separately treated with increasing concentrations $(2,4,20,40 \mu \mathrm{M})$ of nucleozin at RT for $1 \mathrm{~h}$. (D) Aggregation curves obtained by densitometric analysis of separately treated NP (closed circles) and NP-GFP (closed squares) with increasing concentrations of nucleozin at RT for $1 \mathrm{~h}$. The fluorescent supernatants of NP-GFP (open squares) were also quantified by fluorimetry ( $n=3$, see Table S2 in ESI $\dagger$ ). 
unaffected by nucleozin and hence was used as a control in the densitometric method (Fig. 2A). A second experiment was performed where NP $(4 \mu \mathrm{M})$ and NP-GFP $(4 \mu \mathrm{M})$ were analysed separately in the presence of increasing nucleozin concentrations under similar conditions (Fig. 2C). Here again, we observed that the aggregation of NP-GFP is slightly inhibited compared to NP ( $c f$. Fig. 2B and D). Yet NP-GFP was consistently aggregated by nucleozin and can therefore be applied as a reporter for NP aggregation. The $F_{\text {sol }}$ of NP-GFP for each nucleozin concentration was quantified by fluorimetry and reflected the densitometric data (Fig. 2D). A slightly higher background was observed with the fluorimetric method but saturation curves were otherwise identical. The data suggests that the aggregation of NP in the fusion protein is slightly inhibited by the presence of GFP, probably through either a kinetic and/or steric effect. The absence of any ligand-induced effect on GFP or control indicated the specificity and sensitivity of the assay with partially purified or mixtures of proteins. Together, these results validate the use of NP-GFP aggregation based assay for drug screening and resistance profiling studies. Finally, a kinetic evaluation of the assay demonstrated that $1 \mathrm{~h}$ incubation with nucleozin was optimal for fluorimetric quantitation of aggregation and assay reproducibility but that longer incubation times could be used if needed (Fig. S1, ESI $\dagger$ ).

\section{Validation of NP-GFP aggregation assay with anti-NP chicken antibody}

In a next step, we investigated the potential of the NP-GFP aggregation assay to be applied for the screening of biologics promoting aggregation of NP, such as NP-specific antibodies. Recombinant NP expressed in E. coli has previously been shown to efficiently elicit cross-protective immunity in mice. ${ }^{52}$ Here, NP was expressed in E. coli and purified by Ni-affinity chromatography. The purified proteins were then used to immunise chickens. The anti-NP chicken IgYs were purified from the egg yolks of immunised chickens (Fig. 3A) and analysed by immunoblotting (Fig. 3B). The anti-NP IgY fraction obtained showed a strong and specific reactivity against our recombinant H5N1 NP as well as a viral H1N1 NP. Additionally, the Dengue virus envelope protein (EDIII) was produced to serve as a negative control and for the production of a non-specific IgY fraction that was used as a negative control. No reactivity was observed with the negative control (EDIII, $15 \mathrm{kDa}$ ). The negative control antiEDIII IgY showed no reactivity against our recombinant H5N1 NP.

Next, we investigated the effect of increasing concentrations $(0.625-30 \mu \mathrm{M})$ of IgY on NP-GFP $(0.5 \mu \mathrm{M})$. After $30 \mathrm{~min}$ incubation at room temperature, the extent of NP-GFP agglutination was determined after centrifugation and SDS-PAGE. Agglutination of NP-GFP was observed at concentrations starting from $5 \mu \mathrm{M}$ anti-NP IgY, while no agglutination was caused by anti-EDIII IgY at any tested concentration (Fig. 3C). Qualitative assessment of the protein pellets after centrifugation and UV transillumination confirmed these findings (data not shown). The faint bands observed in the absence of IgY represent background levels of NP-GFP aggregation (Fig. 3C).
Fluorimetric quantitation of the reaction supernatants further confirmed these observations (Fig. 3D).

Antibody-mediated agglutination is dependent on both the concentrations of antibody and antigen, i.e. NP-GFP. We investigated the effect of increasing concentrations of NP-GFP against a dilution series of IgY to demonstrate that antibodymediated agglutination was indeed responsible for aggregation of NP-GFP (Fig. 3E). As expected, when higher concentrations of NP-GFP were used a proportionally higher concentration of NPspecific IgY was needed to induce NP-GFP agglutination. Together, these results validate the use of the NP-GFP aggregation assay for the quantitative detection of NP-specific antibodies.

\section{Discussion}

Nucleozin and its analogues are a new class of promising influenza antivirals. They show potent antiviral activity in cell culture and mouse model experiments due to their ability to aggregate NP. In spite of the conserved nature of NP, some of these aggregating compounds were inactive against NP variants harbouring particular mutations. ${ }^{19-22}$ The simplicity and speed of our NP-GFP aggregation assay offers the much needed technology to fast-track the screening and development of new compounds with similar modes of action. Our NP-GFP aggregation assay is an attractive alternative to the recently developed fluorescence quenching assay ${ }^{47}$ as it can be applied with partially purified proteins, eliminating the need for elaborate protein purification. The GFP domain was not subject to aggregation which demonstrates that nucleozin is very specific. GFP can therefore be used as a control protein, i.e. if GFP aggregation is induced by a compound it would be classified as non-specific. The variant of GFP (GFPuv) used in this study is thermostable in a pH range between 5 and $12 .^{53}$ This broad $\mathrm{pH}$ range can be useful to study the effect of $\mathrm{pH}$ on ligand-induced aggregation of NP.

We anticipate that the assay could be translated into a highthroughput format through the replacement of the centrifugation step by a filtration step and the use of appropriate liquid handling robotics. Moreover, both induction and inhibition of protein aggregation can be monitored using this assay. Further applications of this assay format could therefore be extended to study aggregation-prone proteins such as prions, Alzheimer's $A \beta$ and tau. Limiting protein aggregation is a key issue encountered across all stages of the biopharmaceutical formulation and drug development process. This assay could be applied to monitor buffer-induced and undesirable protein aggregation.

The newly developed anti-NP IgY demonstrated specific affinities against both recombinant H5N1 and viral H1N1 NP. This cost-effective, high-yield source of anti-NP IgY has potential for large-scale production and development of therapeutic antibodies. The NP-GFP agglutination induced by the new antiNP IgY was concentration-dependent and demonstrated that the assay could be applied to its quantitation. Hence, it could also be used as a simple screening assay across multiple hosts (i.e. humans, birds, pigs) and multiple influenza subtypes. 


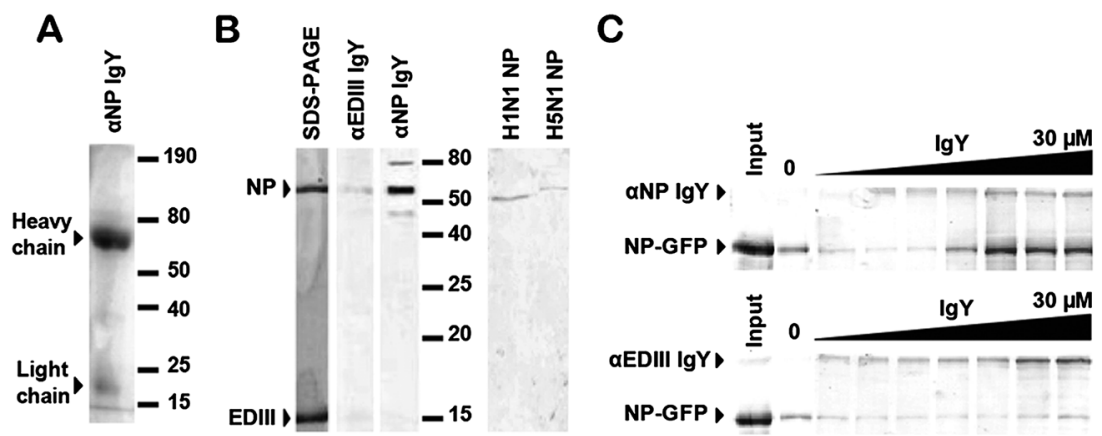

D

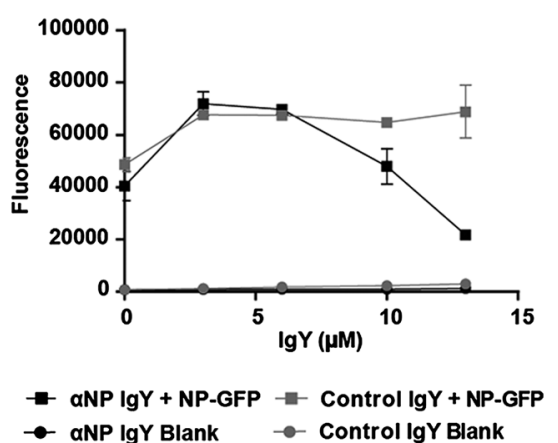

E

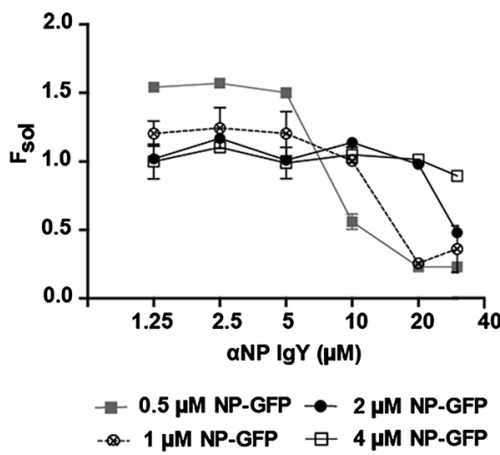

Fig. 3 IgY-induced NP-GFP agglutination. (A) SDS-PAGE of IgY purified from a chicken immunized with NP. (B) Immunoreactivity of anti-NP IgY probed against $3.4 \mu \mathrm{g}$ of NP and $5.6 \mu \mathrm{g}$ EDIII (left panel). Immunoreactivity against $4 \mathrm{ng}$ each of viral H1N1 NP and recombinant H5N1 NP (right panel). (C) NP-GFP agglutination with increasing concentrations $(0.625,1.25,2.5,5,10,20,30 \mu \mathrm{M}$ ) of anti-NP IgY (top panel) and of anti-EDIII IgY (bottom panel). Input: initial amount NP-GFP added to each reaction. Protein bands are revealed by silver staining after SDS-PAGE under non-reducing conditions. Representative gel $(n=3)$. (D) Residual NP-GFP fluorescence in the reaction supernatants after agglutination with increasing concentrations $(3,6,10,13 \mu \mathrm{M})$ of anti-NP or of anti-EDIII (negative control) IgY ( $n=2)$. (E) Concentrationdependent NP-GFP agglutination with increasing concentrations of NP-GFP and anti-NP IgY. Residual fluorescence was measured in the reaction supernatants and expressed in soluble fractions $\left(F_{\text {sol }}\right)(n=2)$.

Current methods for epidemiological surveillance of influenza in avian species rely on serological and egg yolk testing via virus isolation, reverse transcriptase-PCR, blocking enzyme-linked immunosorbent assay, agar gel immunodiffusion test and latex agglutination tests. ${ }^{54-56}$ Comparatively, the NP-GFP agglutination assay offers a fast, simple and low-cost screening alternative for both serum (data not shown) and egg-derived IgY. Screening egg yolks has been found to be a more cost-effective method for poultry surveillance ${ }^{57}$ and our data demonstrate the capability of our assay for testing purified IgY from egg yolk. Furthermore, recent strategies of vaccine development are based on eliciting NP antibodies. In a recent phase I clinical trial, modified vaccinia virus Ankara (MVA) vector expressing influenza NP and matrix protein 1 (MVA-NP + M1) elicited a safe and immunogenic response in humans. ${ }^{58}$ Here, the NP-GFP assay could be used to measure antibody titre post-vaccination and evaluate vaccine efficacies.

In conclusion, the NP-GFP assay is simple, rapid and versatile as it can be applied to the screening of drugs and antibodies targeting NP. The ligand-induced aggregation of NP can be accurately measured irrespective of its binding site. The assay requires minimal protein purification and offers sensitive quantitation even in protein mixtures, which is an advantage when monitoring the effect of biologics such as antibodies. Finally, with its flexibility, we believe that NP-GFP aggregation assay will be a versatile tool for both influenza research and the development of new NP-targeting antivirals.

\section{Acknowledgements}

This work was supported by a grant from the Queensland Smart Futures Fund (NIRAP, Australia).

\section{References}

1 A. M. Thayer, Chem. Eng. News, 2009, 87, 15-26.

2 P. Preziosi, Expert Opin. Pharmacother., 2011, 12, 15231549.

3 M. Hedlund, J. L. Larson and F. Fang, Viruses, 2010, 2, 17661781.

4 D. Butler, Nature, 2009, 459, 14-15.

5 S. B. Graitcer, L. Gubareva, L. Kamimoto, S. Doshi, M. Vandermeer, J. Louie, C. Waters, Z. Moore, K. Sleeman, M. Okomo-Adhiambo, S. A. Marshall, K. St George, C. Y. Pan, J. M. LaPlante, A. Klimov and A. M. Fry, Emerging Infect. Dis., 2011, 17, 255-257.

6 J. E. Morlighem, S. Aoki, M. Kishima, M. Hanami, C. Ogawa, A. Jalloh, Y. Takahashi, Y. Kawai, S. Saga, E. Hayashi, T. Ban, S. Izumi, A. Wada, M. Mano, M. Fukunaga, Y. Kijima, M. Shiomi, K. Inoue, T. Hata, Y. Koretsune, K. Kudo, 
Y. Himeno, A. Hirai, K. Takahashi, Y. Sakai-Tagawa, K. Iwatsuki-Horimoto, Y. Kawaoka, Y. Hayashizaki and T. Ishikawa, PLoS One, 2011, 6, e18956.

7 D. A. Kaminski and F. E. Lee, Front Immunol., 2011, 2, 76. 8 A. Portela and P. Digard, J. Gen. Virol., 2002, 83, 723-734.

9 L. L. Shu, W. J. Bean and R. G. Webster, J. Virol., 1993, 67, 2723-2729.

10 A. K. Ng, J. H. Wang and P. C. Shaw, Sci. China, Ser. C: Life Sci., 2009, 52, 439-449.

11 H. Hayashida, H. Toh, R. Kikuno and T. Miyata, Mol. Biol. Evol., 1985, 2, 289-303.

12 J. Bahl, D. Vijaykrishna, E. C. Holmes, G. J. Smith and Y. Guan, Virology, 2009, 390, 289-297.

13 J. Xu, C. T. Davis, M. C. Christman, P. Rivailler, H. Zhong, R. O. Donis and G. Lu, PLoS One, 2012, 7, e38665.

14 M. Fourment, J. T. Wood, A. J. Gibbs and M. J. Gibbs, Mol. Phylogenet. Evol., 2010, 56, 526-535.

$15 \mathrm{~J} . \mathrm{Xu}, \mathrm{M}$. C. Christman, R. O. Donis and G. Lu, Infect., Genet. Evol., 2011, 11, 2125-2132.

16 N. Sukeno, Y. Otsuki, J. Konno, N. Yamane, T. Odagiri, J. Arikawa and N. Ishida, Tohoku J. Exp. Med., 1979, 128, 241-249.

17 M. W. LaMere, H. T. Lam, A. Moquin, L. Haynes, F. E. Lund, T. D. Randall and D. A. Kaminski, J. Immunol., 2011, 186, 4331-4339.

18 M. J. Amorim, R. Kao and P. Digard, J. Virol., 2013, 87, 46944703.

19 R. Y. Kao, D. Yang, L. S. Lau, W. H. Tsui, L. Hu, J. Dai, M. P. Chan, C. M. Chan, P. Wang, B. J. Zheng, J. Sun, J. D. Huang, J. Madar, G. Chen, H. Chen, Y. Guan and K. Y. Yuen, Nat. Biotechnol., 2010, 28, 600-605.

20 C. Y. Su, T. J. Cheng, M. I. Lin, S. Y. Wang, W. I. Huang, S. Y. Lin-Chu, Y. H. Chen, C. Y. Wu, M. M. Lai, W. C. Cheng, Y. T. Wu, M. D. Tsai, Y. S. Cheng and C. H. Wong, Proc. Natl. Acad. Sci. U. S. A., 2010, 107, 19151-19156.

21 S. W. Gerritz, C. Cianci, S. Kim, B. C. Pearce, C. Deminie, L. Discotto, B. McAuliffe, B. F. Minassian, S. Shi, S. Zhu, W. Zhai, A. Pendri, G. Li, M. A. Poss, S. Edavettal, P. A. McDonnell, H. A. Lewis, K. Maskos, M. Mortl, R. Kiefersauer, S. Steinbacher, E. T. Baldwin, W. Metzler, J. Bryson, M. D. Healy, T. Philip, M. Zoeckler, R. Schartman, M. Sinz, V. H. Leyva-Grado, H. H. Hoffmann, D. R. Langley, N. A. Meanwell and M. Krystal, Proc. Natl. Acad. Sci. U. S. A., 2011, 108, 15366-15371.

22 H. Cheng, J. Wan, M. I. Lin, Y. Liu, X. Lu, J. Liu, Y. Xu, J. Chen, Z. Tu, Y. S. Cheng and K. Ding, J. Med. Chem., 2012, 55, 2144-2153.

23 S. L. Epstein, W. P. Kong, J. A. Misplon, C. Y. Lo, T. M. Tumpey, L. Xu and G. J. Nabel, Vaccine, 2005, 23, 5404-5410.

24 T. M. Fu, L. Guan, A. Friedman, T. L. Schofield, J. B. Ulmer, M. A. Liu and J. J. Donnelly, J. Immunol., 1999, 162, 41634170.

25 J. P. Tite, C. Hughes-Jenkins, D. O'Callaghan, G. Dougan, S. M. Russell, X. M. Gao and F. Y. Liew, Immunology, 1990, 71, 202-207.
26 J. B. Ulmer, T. M. Fu, R. R. Deck, A. Friedman, L. Guan, C. DeWitt, X. Liu, S. Wang, M. A. Liu, J. J. Donnelly and M. J. Caulfield, J. Virol., 1998, 72, 5648-5653.

27 D. M. Carragher, D. A. Kaminski, A. Moquin, L. Hartson and T. D. Randall, J. Immunol., 2008, 181, 4168-4176.

28 M. W. Lamere, A. Moquin, F. E. Lee, R. S. Misra, P. J. Blair, L. Haynes, T. D. Randall, F. E. Lund and D. A. Kaminski, J. Virol., 2011, 85, 5027-5035.

29 R. Patterson, J. S. Youngner, W. O. Weigle and F. J. Dixon, J. Immunol., 1962, 89, 272-278.

30 H. Hatta, K. Tsuda, S. Akachi, M. Kim and T. Yamamoto, Biosci., Biotechnol., Biochem., 1993, 57, 450-454.

31 Z. W. Jaradat and R. R. Marquardt, Food Agric. Immunol., 2000, 12, 263-272.

32 E. Nilsson, J. Stalberg and A. Larsson, Br. Poult. Sci., 2012, 53, 42-46.

33 M. Shimizu, H. Nagashima, K. Sano, K. Hashimoto, M. Ozeki, K. Tsuda and H. Hatta, Biosci., Biotechnol., Biochem., 1992, 56, 270-274.

34 J. Kovacs-Nolan and Y. Mine, Avian Poultry Biol. Rev., 2004, 15, 25-46.

35 A. Sesarman, S. Mihai, M. T. Chiriac, F. Olaru, A. G. Sitaru, J. M. Thurman, D. Zillikens and C. Sitaru, Br. J. Dermatol., 2008, 158, 463-471.

36 J. Kovacs-Nolan and Y. Mine, Annu. Rev. Food Sci. Technol., 2012, 3, 163-182.

37 M. G. Wallach, R. J. Webby, F. Islam, S. Walkden-Brown, E. Emmoth, R. Feinstein and K. O. Gronvik, Clin. Vaccine Immunol., 2011, 18, 1083-1090.

38 J. Kovacs-Nolan and Y. Mine, Food Biotechnol., 2004, 18, 39-62. 39 D. Pauly, M. Dorner, X. Zhang, A. Hlinak, B. Dorner and R. Schade, Poult. Sci., 2009, 88, 281-290.

40 R. Schade, E. G. Calzado, R. Sarmiento, P. A. Chacana, J. Porankiewicz-Asplund and H. R. Terzolo, ATLA, Altern. Lab. Anim., 2005, 33, 129-154.

41 H. H. Nguyen, T. M. Tumpey, H. J. Park, Y. H. Byun, L. D. Tran, V. D. Nguyen, P. E. Kilgore, C. Czerkinsky, J. M. Katz, B. L. Seong, J. M. Song, Y. B. Kim, H. T. Do, T. Nguyen and C. V. Nguyen, PLoS One, 2010, 5, e10152.

42 J. Wen, S. Zhao, D. He, Y. Yang, Y. Li and S. Zhu, Antiviral Res., 2012, 93, 154-159.

43 I. Morin and P. M. Schaeffer, Anal. Biochem., 2012, 420, 121126.

44 N. J. Moreland, P. Susanto, E. Lim, M. Y. Tay, R. Rajamanonmani, B. J. Hanson and S. G. Vasudevan, Int. J. Mol. Sci., 2012, 13, 2618-2635.

45 R. Rajamanonmani, C. Nkenfou, P. Clancy, Y. H. Yau, S. G. Shochat, S. Sukupolvi-Petty, W. Schul, M. S. Diamond, S. G. Vasudevan and J. Lescar, J. Gen. Virol., 2009, 90, 799-809.

46 D. B. Dahdah, I. Morin, M. J. Moreau, N. E. Dixon and P. M. Schaeffer, Chem. Commun., 2009, 3050-3052.

47 H. C. Hung, C. L. Liu, J. T. Hsu, J. T. Horng, M. Y. Fang, S. Y. Wu, S. H. Ueng, M. Y. Wang, C. W. Yaw and M. H. Hou, Anal. Chem., 2012, 84, 6391-6399.

48 D. Pauly, P. A. Chacana, E. G. Calzado, B. Brembs and R. Schade, J. Visualized Exp., 2011, e3084. 
49 M. J. Moreau, I. Morin and P. M. Schaeffer, Mol. BioSyst., 2010, 6, 1285-1292.

50 A. Fomsgaard, M. A. Freudenberg and C. Galanos, J. Clin. Microbiol., 1990, 28, 2627-2631.

51 M. J. J. Moreau, I. Morin, S. P. Askin, A. Cooper, N. J. Moreland, S. G. Vasudevan and P. M. Schaeffer, RSC Adv., 2012, 2, 11892-11900.

52 B. Huang, W. Wang, R. Li, X. Wang, T. Jiang, X. Qi, Y. Gao, W. Tan and L. Ruan, Virol. J., 2012, 9, 322.

53 T. C. V. Penna, M. Ishii, J. S. Kunimura and O. Cholewa, Appl. Biochem. Biotechnol., 2005, 121, 501-527.

54 J. D. Brown, M. P. Luttrell, R. D. Berghaus, W. Kistler, S. P. Keeler, A. Howey, B. Wilcox, J. Hall, L. Niles, A. Dey, G. Knutsen, K. Fritz and D. E. Stallknecht, J. Wildl. Dis., 2010, 46, 896-911.
55 M. Horie, H. Ogawa, K. Yamada, A. Hara, V. N. Bui, S. S. Awad, R. Yoshikawa, M. Mase, K. Tsukamoto, S. Yamaguchi, K. Nakamura and K. Imai, J. Virol. Methods, 2009, 161, 259-264.

56 J. R. Beck, D. E. Swayne, S. Davison, S. Casavant and C. Gutierrez, Avian Dis., 2003, 47, 1196-1199.

57 O. M. Jeong, M. C. Kim, H. M. Kang, G. W. Ha, J. S. Oh, J. E. Yoo, C. H. Park, J. S. Kwon, M. R. Pack, H. R. Kim, Y. J. Kim, J. H. Kwon and Y. J. Lee, Vet. Microbiol., 2010, 144, 287-292.

58 P. J. Lillie, T. K. Berthoud, T. J. Powell, T. Lambe, C. Mullarkey, A. J. Spencer, M. Hamill, Y. Peng, M. E. Blais, C. J. Duncan, S. H. Sheehy, T. Havelock, S. N. Faust, R. L. Williams, A. Gilbert, J. Oxford, T. Dong, A. V. Hill and S. C. Gilbert, Clin. Infect. Dis., 2012, 55, 19-25. 